\title{
Hypnosis as an intervention in pain management: A brief review
}

\author{
BRENDA KING ${ }^{2}$, MICHAEL NASH ${ }^{1}$, \\ DAVID SPIEGEL ${ }^{2}$ AND \\ KENNETH JOBSON ${ }^{3}$ \\ ${ }^{1}$ University of Tennessee; ${ }^{2}$ Stanford University \\ School of Medicine; and ${ }^{3}$ University of \\ Tennessee School of Medicine, USA
}

\section{Correspondence Address}

Brenda J. King, PhD, University of Washington, Box 356560, Department of Psychiatry and Behavioral Science, Seattle, WA 98195, USA.

Tel: (203)-368-7078

E-mail:bjking@utk.edu or

bjking@u.washington.edu

Received 1 June 1999; revised 20 December 2000; accepted for publication 22 December 2000
While there is a consensus that psychosocial factors play an important role in the experience of pain, clinical interventions for acute and chronic pain remain primarily biomedical. This pattern persists despite a body of recent empirical work supporting the effectiveness of a number of behavioral and relaxation interventions for these problems. One of these interventions is hypnosis. We briefly discuss hypnotic analgesia and describe how hypnosis can be integrated into biomedical treatment for acute and chronic pain. Special attention is given to indications and contraindications, preparation of patients, and technique. (Int J Psych Clin Pract 2001; 5: 97-101)
Keywords hypnosis acute pain hypnotic assessment chronic pain

clinical studies n 1966 a US National Institute of Health Technology Assessment Panel noted that despite the wide variety of surgical and pharmaceutical options available, certain types of pain continue to be a significant problem. ${ }^{1}$ The experience of pain is a result of the complex interaction between physical sensation and psychosocial factors, with the physical awareness of noxious stimuli markedly influenced by the meaning of the pain for the patient. ${ }^{2-4}$ the NIH further cited hypnosis as one of several viable and effective interventions for alleviating pain in cancer and other chronic pain conditions. Indeed, the property of hypnosis which has the greatest potential for social good resides in the ability of the hypnotizable subjects to radically reduce, or in some cases eliminate, both chronic and acute pain. We here briefly review hypnosis as an important adjunct to the successful management of acute and chronic pain.

\section{THE PROBLEM OF PAIN}

\section{Acute/Respondent Pain}

Acute pain is typically time-limited and associated with impending or actual tissue damage as a result of injury or illness. ${ }^{2}$ The pain is a direct response to tissue damage and also serves as a signal to alter behavior. This type of pain is routinely associated with injury or illness. Medical interventions such as invasive diagnostic procedures, burn treatment, and surgery can also produce this type of acute pain in patients. Even when the tissue trauma is invariant, patients' experience of pain varies widely, depending on constitutional factors (such as their pain threshold) as well as situational factors (proximal anxiety, emotional meaning).

\section{Chronic/Operant Pain}

Chronic pain is more complex in that the sensation of pain may no longer serve an adaptive function, and psychosocial factors frequently exacerbate or maintain the experience beyond what would be expected on the basis of physical findings. ${ }^{5}$ Conditions previously associated with pain, rather than further tissue injury, may trigger attacks: hence the term 'operant'. Low back pain, arthritis, reflex sympathetic dystrophy, fibromyalgia, and phantom limb syndrome are examples of conditions leading to chronic pain. ${ }^{4,5}$

\section{HYPNOSIS AND PAIN}

Despite many options for managing both acute and chronic pain, results continue to be less than satisfactory at times. ${ }^{1}$ 
For some patients, hypnosis can be a valuable tool in the self-management of both acute and chronic pain, yet it is underutilized. ${ }^{6}$ In fact hypnosis has a long history of use in pain treatment, ${ }^{7,8}$ dating back to the 19 th century. For over a century surgical procedures have been (and sometimes still are) performed with hypnosis as the sole source of pain relief. ${ }^{9,10}$

Controlled clinical trials have examined the use of hypnosis across a spectrum of clinical problems related to pain and have shown that the use of hypnotic techniques can be beneficial for some patients. ${ }^{1-19}$ Recently it has been demonstrated that training patients in the use of selfhypnosis significantly reduced their need for sedatives and analgesia when undergoing interventional radiological procedures. $^{11,12}$ Thirty patients undergoing interventional radiological procedures were randomized to either a treatment or a control group. ${ }^{11}$ Those individuals in the treatment group were presented with instructions for selfhypnosis and imagery during initiation of the radiological procedure and were intermittently prompted to use selfhypnosis throughout the procedure. The assigned practitioner also obtained anxiety and pain ratings throughout the procedure. Those individuals in the control group were also assigned a practitioner but interactions were limited to obtaining pain and anxiety ratings. All patients were instructed on the use of 'patient-controlled analgesia', in which they could administer themselves intravenous sedatives and narcotics within a controlled range. Patients in the treatment group reported significantly less pain and anxiety and administered themselves significantly less medication than did those in the control group. Interestingly, the patients in the treatment group also experienced fewer medical complications as a result of the procedures and required less medical staff time immediately after the procedures.

Similar positive effects were found for patients undergoing routine plastic surgery under conscious sedation. ${ }^{15}$ Sixty patients were randomly assigned to either a hypnosis $(n=35)$ or an emotional support condition $(n=25)$. The hypnosis condition consisted of an induction and imagery of a positive life experience during the operative procedure. No suggestions for analgesia were given. The emotional support condition consisted of reassurance, information about the procedures, and conversational distraction during the operative procedure. Patients in the hypnosis condition were found to experience significantly less postoperative anxiety, pain, nausea and vomiting than did patients in the emotional support condition. Use of intraoperative sedatives was also significantly less in the hypnosis condition. Further, patient-perceived control was higher, while patients' interference with procedure was lower with the use of hypnosis. This study provides additional evidence that the use of adjunctive hypnosis during medical procedures can provide improved pain management and reduce post-operative discomfort.

Another recent example of the efficacy of hypnosis as an integrated component of medical care is demonstrated in a report of the positive effects found with patients undergoing orthopedic hand surgery. ${ }^{19}$ Sixty hand-surgery patients were assigned to either the usual treatment, or usual treatment plus hypnosis, in a quasi-experimental design. Two consecutive cohorts were used, rather than random assignment. The control group was run first, followed by the hypnosis group. The hypnotic treatment provided consisted of a standardized induction, with suggestions for relaxation and positive outcome. Those patients assigned to the hypnotic intervention group reported less pain and anxiety, while physician's ratings indicated faster post-operative progress and fewer medical complications. Measurements were obtained at each of the first four post-operative days. The overall multivariate effect size was 0.44 . While the absence of randomization somewhat limits the conclusions that can be drawn from this study, the results are consistent with a growing body of work that provides support for the efficacy of hypnosis in pain management.

Chronic pain provides a significant challenge for healthcare providers, as many factors contribute to the ongoing problem of pain. Nonetheless, some clinical experiments using patients with chronic pain problems have shown that these individuals, too, can benefit from the use of hypnosis. For instance, in one study fibromyalgia patients treated with hypnotic suggestions for reduction of muscle pain and improved sleep experienced greater improvement in symptoms that did comparison patients treated with relaxation and massage. ${ }^{13}$ The group treated with hypnosis also required less medication.

Chronic back pain is a debilitating and costly problem that does not remit for approximately $10 \%$ of patients who develop back pain. ${ }^{20}$ There is evidence that hypnosis can provide some benefits for this challenging group of patients. Fifteen adults with chronic low back pain (mean length of complaints, 4 years) participated in a two-part study which examined hypnotic responsiveness in the face of laboratory-induced pain and then examined the ability of participants to transfer learned skills to their problem of low pack pain. ${ }^{21}$ The participants were self-referred, based upon information sheets provided to their physician or chiropractor. All but one were found to be moderately to highly hypnotizable. The research took place over three 3hour experimental sessions, separated by 1 week. When presented with experimentally induced cold-pressor pain, participants with hypnosis demonstrated a significant reduction in sensory pain and associated distress as compared to those with no hypnosis. The design was an A-B-A design in which each participant served as his or her own control. At the completion of the first experimental session, a discussion was held with each participant to discuss his or her use of hypnosis to manage the experimentally induced pain. They were encouraged to apply these techniques at their own discretion to their low back pain. Over a 4-week period the participants reported significant reductions in pain, improved sleep quality, and improved psychological well-being. This study provides 
support for the efficacy of hypnosis for chronic back pain and suggests that experimental reductions in pain complaints can be generalized to clinical problems.

The question then is: how does hypnosis lead to reduced pain and improved clinical outcomes? Hypnosis is a form of highly focused concentration (absorption), with a relative suspension of perplexed awareness. It enables people to put sensations and thoughts ordinarily processed in the consciousness outside of awareness (dissociation). This property of hypnosis can be used to put pain perception at the periphery of consciousness, or to radically transform the experience of the pain signal. ${ }^{22}$ Further, hypnotized individuals, as a rule, establish a more secure positive alliance with the clinician and are more likely to respond to therapeutic intervention. ${ }^{23}$

Early work tested the notion that the reductions in pain might have nothing to do with hypnosis at all, but may simply reflect the operation of non-specific factors that have modest pain-reducing properties. Relaxation, placebo response, and distraction are all associated with some attenuation of pain report, and all can be a component of any intervention for pain. Could these be the actual active agents that account for hypnotic analgesia? There is now good reason to believe that this is not the case. The mechanism by which hypnosis achieves these effects remains elusive, but research demonstrates fairly conclusively that the mechanism involved is distinct from that for placebo, relaxation, or distraction. ${ }^{24-26}$ Much laboratory research has been devoted to understanding the underlying mechanisms of hypnotic analgesia, using experimentally induced pain (such as cold pressor, ${ }^{21,27}$ ischemic, electrical, $^{28,29}$ pressure $^{30}$ or laser-induced techniques. ${ }^{25}$ It is clear now that people who experience hypnosis more fully and easily derive the most benefit from hypnotic analgesia, with sometimes dramatic improvements. Poorly-hypnotizable subjects, on the other hand, presumably exposed to the same non-specific factors, typically only achieve a very modest effect (akin to what one might expect from placebo).

But beyond this correlational approach, two pivotal experimental studies ruled out the notion that placebo and relaxation could explain hypnotic analgesia. A study conducted at the University of Pennsylvania Experimental Psychiatry laboratories found that for 'low-hypnotizable' subjects, hypnotic analgesia acted like a placebo - these subjects obtained comparable and minimal relief from placebo and hypnotic analgesia conditions. ${ }^{24}$ Further, the extent to which they obtained relief during hypnotic analgesia was correlated with the extent to which they obtained relief from placebo intervention $(r=0.76)$. So far, the placebo hypothesis was looking quite good. In sharp contrast, though, highly hypnotizable subjects achieved a far greater reduction in pain, and their placebo response was near to, or even slightly below, baseline. Importantly, the extent to which these highly hypnotizable subjects experienced relief during hypnosis was unrelated to their placebo response.
In a second study, a similar design was used, except that pain was induced by argon laser, and amplitudes of painevoked potentials were monitored throughout. ${ }^{25}$ The highly hypnotizable group displayed significantly better reductions across conditions, but they responded poorly to placebo. Further, the only condition in which pain-related evoked potentials dropped below baseline was during hypnotic analgesia among highly hypnotizable subjects.

The mechanisms underlying hypnotic analgesia are still not fully understood. Three possibilities have been noted. The model favored by most is that the analgesic effect is centrally mediated, occurring after nociception has reached the higher brain centers. ${ }^{2}$ This would account for the fact that most autonomic responses that routinely accompany pain also occur under conditions of profound hypnotic analgesia. The second possibility is that the operable mechanism is localized more peripherally. But there are problems with this. One would be hard pressed to explain why hypnotic analgesia can be terminated upon cue if the process involved an anti-nociceptive mechanism, and there is compelling evidence that hypnotic analgesia is not mediated by endorphins. ${ }^{31}$ Finally, some believe that it is a combination of central and peripheral mechanisms, with central mechanisms impacting mostly the emotional or 'suffering' component of the pain experience.

\section{HYPNOTIC RESPONSIVENESS}

Although hypnotic intervention can provide some benefit to most patients, the more dramatic improvements take place in patients in pain who are also moderately to highly responsive to hypnotic suggestion. This quality of being highly responsive to hypnosis (highly hypnotizable) is a stable personality trait that does not vary much across situation or time (even decades). In fact, the stability of hypnotic responsiveness as a trait is comparable to that of the results of IQ tests. ${ }^{32}$ Individuals who are highly responsive to hypnotic suggestions will respond most rapidly to hypnotic interventions and obtain the most salient outcomes, regardless of treatment objective. ${ }^{8}$ Lowhypnotizable patients have been shown to benefit from hypnotic techniques also, but are less likely to experience satisfactory analgesia. ${ }^{8}$ Is it possible then to identify those pain patients who are also highly responsive to hypnosis?

Assessing hypnotizability can be accomplished with a number of short scales developed specifically for this purpose.

1. The Hypnotic Induction Profile (HIP) ${ }^{33}$ is a $5-10 \mathrm{~min}$ method of appraising hypnotic responsivity which involves assessing the patient's capacity to look up while closing their eyes. A number of suggestions are then given to the patient to assess their response to brief structured hypnotic experiences.

2. An alternative method for assessing hypnotizability is the Stanford Hypnotic Clinical Scale (SHCS). ${ }^{34}$ This 
scale includes a variety of hypnotic phenomena and requires minimal effort on the clinician's part. As it requires only $20-30 \mathrm{~min}$ to administer it also provides a practical method of assessment.

3. A new alternative for assessing hypnotizability is an interactive computer-administered procedure, which has the advantage of reduced time and effort on the part of the clinician, and renders a comprehensive profile of the patient's abilities. The Computer Assisted Hypnosis Scale ${ }^{35}$ interacts with the patient's responses, taking the subject through a hypnotic induction and 12 test suggestions. Its reliability and validity profiles are comparable to those of comprehensively researched scales. This scale has the advantage of providing information about the patient's subjective responsivity as well as behavioral responsiveness.

\section{TREATMENT APPLICATIONS}

Once a patient has been judged to be suitable for hypnosis, a decision must be made about what type of intervention will be most useful. Hypnosis is a flexible tool that can be utilized within the framework of most psychosocial approaches to pain (such as cognitive-behavioral, psychodynamic, behavior modification).

Techniques can be tailored to fit with the medical staffs treatment philosophy and biomedical protocol. For instance, while individual therapy (one-in-one sessions) is perfectly appropriate for patients, hypnosis can also be successfully incorporated into group work. Spiegel and Bloom $^{14}$ illustrated a typical use of hypnosis for women with Stage 3 metastatic breast cancer. Patients in the treatment group met weekly for a 90-min support group which utilized $5 \mathrm{~min}$ of self-hypnosis as a routine group exercise. Compared to control subjects, the women in the treatment group experienced less pain and suffering during treatment and at 1-year follow-up.

If a patient is trained in how to use self-hypnosis, they will be able to employ the coping skill as needed in daily life without a clinician in attendance. ${ }^{21,36}$ Self-hypnosis can consist of the patient listening to an audiotaped induction and suggestions from the clinician, or (preferably) it can be entirely self-directed. The clinician can work with the patient to develop a method of self-directed induction and suggestion which allows the motivated patient greater control and versatility. Self-hypnosis has been found to be useful both for chronic pain and for situations in which repeated painful experiences are expected (eg, invasive medical procedures such as bone marrow aspirations, obstetrics procedures, wound debridement). ${ }^{18,37,38}$

Of course individual work with the patient allows the patient and clinician to develop more fully suggestions that can be successively tailored to manage the pain experience. Such an approach may allow the experienced clinician to work with patients in whom there has not been time to formally assess hypnotic responsiveness. In an emergency room setting a clinician may find it necessary to incorporate assessment and treatment into a single encounter. ${ }^{38}$ In these cases suggestions are used both to assess the patient's responsiveness and to provide the most relief possible.

Hypnotic interventions will vary, depending on the patient's responses to suggestion. Some highly motivated and highly hypnotizable patients will be able to experience complete ablation of sensation upon direct suggestion. This approach may be successful with only a small segment of the population. Alternatives to suggesting anesthesia include altering the pain sensation so that it is perceived in a less disturbing manner. For example, suggesting an experience of warmth or itching may lead to greater tolerance of pain; and other alternatives include directing attention away from the pain and source of pain by use of imaginal distancing or redirection of attention.

\section{Patient Preparation}

In preparing patients for hypnosis it is important to explore any beliefs or misconceptions they hold regarding hypnosis. ${ }^{35}$ Patients may fear that hypnosis will lead to loss of control. For these patients it may be important to emphasize that hypnosis makes use of innate abilities and that the therapist serves as a guide in helping the patient to creatively use concentration and imaginal abilities they already have to gain control over pain. Use of self-hypnosis is another method of allowing the patient the greatest sense of control. The point here is to communicate to the patient that hypnosis will enable them to acquire more, not less, control of their body. Another misconception patients sometimes hold is that the use of hypnosis implies that their discomfort is "all in their head"; the therapist communicates that he/she knows the pain is a real experience. In fact, mentally healthy individuals are more likely to be able to utilize hypnosis, no matter what its origin. ${ }^{36}$

\section{SUMMARY}

Hypnotic interventions can complement pharmacological pain management and lead to decreased use of medica-

\section{KEY POINTS}

- Hypnosis is a viable and effective adjunctive intervention for managing pain

- The mechanisms involved in hypnotic analgesia are distinct from those in placebo, relaxation, or distraction

- Individuals who are highly responsive to hypnotic suggestions will respond most rapidly to hypnotic interventions and obtain the most benefits 
tion. ${ }^{11}$ Hypnosis is a safe, noninvasive and reasonably effective component of pain management that can lead to amelioration of both the sensory and affective components of pain for some patients. Hypnosis is properly used only as an adjunctive component in an established comprehensive pain management protocol. While hypnosis will not work for every patient, it provides an additional option for managing pain and complements traditional medical approaches to pain management.

\section{REFERENCES}

1. National Institute of Health (NIH) (1996) NIH Technology Assessment Panel on Integration of Behavioral and Relaxation Approaches into Treatment of Chronic Pain and Insomnia. JAMA 276: $313-8$.

2. Melzack R, Wall PD (1982) The Challenge of Pain. New York: Basic Books.

3. Fernandez E, Turk DC (1982) Sensory and affective components of pain: separation and synthesis. Psychol Bull 112: 20517.

4. Flor H, Birbaumer N, Turk DC (1990) The psychobiology of chronic pain. Adv Behav Res Ther 12: 47-84.

5. Turk DC, Rudy TE (1987) Towards a comprehensive assessment of chronic pain patients. Behav Res Ther 25: 237-49.

6. Holyroyd J (1996) Hypnosis treatment of clinical pain: understanding why hypnosis is useful. Internat J Clin Exper Hypnosis 44: $33-51$.

7. Barber J (ed) (1996). Hypnosis and Suggestion in the Treatment of Pain. New York: Norton.

8. Hilgard ER, Hilgard JR (1994) Hypnosis in the Relief of Pain (revised edn) New York: Brunner/Mazel.

9. Kubiak RV (1983) Hypnosis: Anesthetic agent in major surgery: A case report. J Med Hypnoanal 4: 46-8.

10. Rausch V (1980) Cholecystectomy with self-hypnosis. Am J Clin Hypnosis 22: 124-9.

11. Lang E, Joyce J, Spiegel D et al (1996) Self-hypnotic relaxation during interventional radiological procedures: effects on pain perception and intravenous drug use. Internat J Clin Exper Hypnosis 44: $106-119$.

12. Lang E, Berbaum KS (1997) Educating interventional radiology personnel in nonpharmacologic analgesia. Academy Radiol 4: $753-7$.

13. Hannen HCM, Hoenderdos HTW, Van Romunde LKJ et al (1991) Controlled trials of hypnotherapy in the treatment of refractory fibromyalgia. I Rheumatol 18: 72-75.

14. Spiegel D, Bloom JR (1983) Pain in metastatic breast cancer. Cancer 52: $341-5$

15. Faymonville ME, Mambourg PH, Joris J et al (1997) Psychological approaches during conscious sedation. Hypnosis versus stress reducing strategies: a prospective randomized study. Pain 73: $361-7$.

16. Patterson DR, Ptacek JT (1997) Baseline pain as a moderator of hypnotic analgesia for burn injury treatment. J Consult Clin Psychol 65: 60-67.

17. Moore LE, Wiesner SL (1996) Hypnotically-induced vasodilation in the treatment of repetitive strain injuries. Am J Clin Hypnosis 39: $97-104$.

18. Patterson DR, Sharar S (1997) Treating pain in patients with severe burn injuries. Adv Med Psychother 9: 55-71.

19. Magaly HM, Burnett KF, Oullete EA et al (1999) Medical hypnosis and orthopedic hand surgery: pain perception, postoperative recovery, and therapeutic comfort. Internat J Clin Exper Hypnosis 47: 144-61.

20. Nachmenson A (1982) the natural course of low back pain. In: White AA, Gordan SL (eds) Symposium on Idiopathic Low Back Pain. St. Louis, MO: Mosby, 46-51.
21. Crawford HJ, Knebel T, Kaplam L et al (1998) Hypnotic analgesia: 1. Somatosensory event-related potential changes to noxious stimuli. 2. Transfer learning to reduce chronic low back pain. Internat J Clin Exper Hypnosis 46: 92-132.

22. Price DD, Barber J (1987) A quantitative analysis of factors that contribute to the efficacy of hypnotic analgesia. J Abnormal Psychol 96: 46-51.

23. Wadden TA, Anderton CH (1982) The clinical use of hypnosis. Psychol Bull 91: 215-43.

24. McGlashen TH, Evans FJ, Orne MT (1984) The nature of hypnotic analgesia and the placebo response to experimental pain. Psychosom Med 31: 227-46.

25. Zachariae R, Bjerring P (1994) Lasar-induced pain-related brain potentials and sensory pain ratings in high and low hypnotizable subjects during hypnotic suggestions of relaxation, dissociated imagery, focused analgesia, and placebo. Internat $J$ Clin Exper Hypnosis 42: 56-80.

26. Farthing GW, Venturino M, Brown SW et al (1997) Internal and external distraction in the control of cold-pressor-pain as a function of hypnotizability. Internat J Clin Exper Hypnosis 45: 433-46.

27. Barber TX, Hahn KW (1962) Physiological and subjective responses to pain producing stimulation under hypnotically suggested and working-imagined "imagined" analgesia. $J$ Personality Social Psychol 65: 411-8.

28. Sharev U, Tal M (1989) Masseter inhibitory periods and sensations evoked by electrical tooth-pulp stimulation in subjects under hypnotic analgesia. Brain Res 479: 247-54.

29. Kiernan BD, Dane JR, Phillips LH, Price DD (1995) Hypnoanalgesia reduces R-III nocioceptive reflex: Further evidence concerning the multifactorial nature of hypnotic analgesia. Pain 60: 39-47.

30. Chaves JF, Barber TX (1974) Cognitive strategies, experimenter modeling and expectation in the attenuation of pain. J Abnormal Psychol 83: 356-63.

31. Spiegel D, Albert A (1983) Naloxone fails to reverse hypnotic alleviation of chronic pain. Psychopharmacol 81: 140-3.

32. Piccionne C, Hilgard ER, Zimbardo PG (1989) On the degree of stability of measured hypnotizability over a 25 -year period. $J$ Personality Social Psychol 56: 289-95.

33. Spiegel H (1974) Manual for Hypnotic Induction. New York: Soni Medica.

34. Morgan AH, Hilgard JR (1978-79) The Stanford Hypnotic Clinical Scale for Adults. Am J Hypnosis 21: 134-47.

35. Grant CD, Nash MR (1995) The computer assisted hypnosis scale: standardization and forming of a computer-assisted measure of hynotic ability. Psychol Assess 7(1): 49-58.

36. Spiegel D (1994) Hypnosis. In: Hales RE, Yudofsky SC, Talbott JE (eds) American Psychiatric Press Textbook of Psychiatry. Washington, DC: American Psychiatric Press, 1142-1158.

37. Chaves JF (1992) Hypnosis in pain management. In: From E, Nash M (eds) Contemporary Hypnosis and Research. New York: Guilford Press.

38. Spira JL, Spiegel D (1992) Hypnosis and related techniques in pain management. Hospice J 8: 89-119. 